\title{
Microfluidic platforms for the detection of disease biomarkers
}

\begin{abstract}
Biomarkers, which can be obtained from different sources including blood, tissues, body fluids, and urine, can be evaluated to indicate normal, pathogenic, or therapeutic response to biological system. Early detection of biomarkers not only prevent the spread of infectious disease but also drastically decrease the death rate of people suffering from different diseases, especially in rural areas where financial resources are limited. Low cost microfluidic technologies have applications in human health diagnosis, food safety, and environmental analysis. This article briefly reviews different kinds of microfluidic platforms including paper, polymer, and hybrid platforms as a microfluidic biosensor for the detection of disease biomarkers. It also discusses the current limitations and future prospects.
\end{abstract}

Volume I Issue I - 2017

\author{
Sujata Chalise,' Anoj Timilsina² \\ 'Pokhara University, Nepal \\ ${ }^{2}$ Department of Microbiology, Pokhara University, Nepal
}

Correspondence: Sujata Chalise, School of Health and Allied Sciences, Pokhara University, Lekhnath-12, Kaski, Nepal,Tel

+9779840010908, Email csuzz.sc@gmail.com

Received: January 18, 2017| Published: February 15, 2017

Keywords: biomarkers, infectious diseases, microfluidic technology, biosensors

\section{Introduction}

Biomarkers such as proteins, lipids, metabolites, circulating DNA and tumor cells, mRNA expression profiles, and electrical signals are the physiological signals that changes in response to the disease conditions and can be evaluated to indicate the normal biological process, pathogenic condition or the response of body to therapeutic drugs. ${ }^{1}$ These biomarkers can be obtained from different sources including blood, urine, tissues, and other body fluids. Acute infectious diseases are caused by varieties of pathogens including bacteria, fungi, parasites, helminths, and viruses. ${ }^{2-5}$ Infectious diseases have been the leading cause of death and disability rates through centuries. In addition to infectious diseases cancer is also the leading cause of death. According to World Health Organization, 8.2 million deaths were reported in 2012. ${ }^{6}$ In addition, 8 million new cases of cancer, 5.3 million-cancer death and 15.6 million 5-year prevalence cancer cases were reported in under developed countries and low-resource settings. ${ }^{6}$ More than $95 \%$ of death resulting due to infectious disease occurs in underdeveloped countries where there is lack of proper diagnosis as well as treatment due to difficulty in getting healthcare infrastructures. ${ }^{7}$

Biomarkers for infectious diseases and cancer are usually diagnosed by exhausting laboratory tests including immunoassay, western blotting, polymerase chain reaction, flow cytometry, and wide range of other techniques. ${ }^{8-10}$ Most of these detection techniques are highly complicate and time consuming, and require specialized, bulky, and expensive equipments. In addition, these techniques consume large volume of expensive reagents and limited samples. These kinds of detection techniques cannot be used in resource poor settings and developing countries.

According to $\mathrm{WHO}$, for the test to be appropriate for under developing countries, different characteristics has to be satisfied which has been abbreviated as ASSURED. It includes, 1) Affordable to everyone, 2) Sensitive, 3) Specific, 4) User-friendly, 5) Rapid treatment and robust use, 6) Equipment-free, and 8) Delivered to those who need it. ${ }^{11}$ Therefore, for the detection of these biomarkers in resource-limited settings, a simple, portable, and low-cost diagnostic device that is highly sensitive and specific is a must. ${ }^{12}$

\section{Discussion}

\section{Microfluidic devices}

Microfluidic lab-on-a-chip (LOC), a science of manipulating very small amounts of fluids within a micro-scale device, can be produced by micro fabrication technique. It is a growing field in science and technology, since its advent in the 1990s. It possesses several features including low-cost, simple and fast analysis, low reagent consumption, high capillary electrophoresis separation efficiency, precise control over the fluid behavior, and high efficiency and sensitivity because of its high surface to volume ratio and micro liter volume of micro channel. These devices with inherent miniaturization, integration, and automation can analyze complex biological fluids for healthcare applications. ${ }^{13,14}$ Different varieties of microfluidic platforms have been used for the detection of disease biomarkers including silicon and glass, polymers, paper, and hybrid devices. The choice of microfluidic platform depends upon several factors including, research application, detection technique, cost, availability of micro-fabrication tools, and thermal and chemical properties. Micro fabrication technologies developed from microelectronics field involved glass, quartz, and silicon as microfluidic platform in early days. These materials are more expensive, lack rapid prototyping, and have low biocompatibility. In addition, they have stringent requirement of clean room facility and are not suitable for point of care (POC) settings.

\section{Paper-based devices}

Paper, a thin sheet of porous hydrophilic material produced by pressing together cellulosic or nitrocellulose fibers can transport liquid through capillary effect, without external force and its fabrication does not require clean room facility. Its high surface to volume ratio provided by macro-porous structure improves detection limit for colorimetric method and also improves the immobilization of proteins, enzymes, biosensors, and DNA biomarkers. ${ }^{9}$ The ability to store reagents in active form within the 3D structure for months at room temperature is also an important feature. ${ }^{15}$ These paper-based devices can be fabricated either by construction of hydrophobic barriers or two-dimensional cutting. 
Hydrophobic barriers can be constructed in the hydrophilic paper matrix so that the reagents and analytes can flow in a certain path and mixing or spreading across the surrounding paper surface can be prevented. Fabrication of paper-based device through construction of hydrophobic barriers can be done by different methods including, fast photolithography, wax-based fabrication techniques (wax screenprinting, wax dipping, and wax printing), printing photolithography, polydimethylsiloxane (PDMS) printing, saline ultra-violet ozone (UV/O3) patterning, flexographic printing, and alkenyl ketene dimer (AKD) printing. ${ }^{1,16}$

Paper-based assays can be done in different ways including dipstick assays, lateral flow assays and microfluidic paper-based analytical devices. ${ }^{17}$ Microfluidic paper-based analytical devices ( $\mu$ PADs) was pioneered by Dr. White sides group. ${ }^{18}$ Application of paper-based microfluidic platforms includes, human health diagnosis, nucleic acid analysis, cellular analysis, and food safety analysis. ${ }^{18}$ Different approaches have been used for the detection using paperbased devices including colorimetric detection, chemiluminescence detection, electrochemical detection, and fluorescence detection. ${ }^{19}$ Paper-based devices have been used for protein analysis (infectious diseases and cancer), nucleic acid analysis (dengue virus, meningitis), and cellular analysis (bacteria and cancer cells) and other analytes like glucose and urobilinogen. In addition it has been used for the detection of pesticides and food borne pathogens. ${ }^{8,20}$

\section{Polymer-based devices}

Fabrication of polymer devices is simple and does not require hazardous etching reagents. Along with paper some polymer such as PDMS, and thermoplastics have been widely used as the substrate for microfluidic devices. PDMS is optically transparent and elastic, easy to fabricate, gas permeable, biocompatible and can seal with itself and other materials by exposing it to air plasma. It has limitations likes swelling in organic solvents, low mechanical and temperature resistance, and hydrophobicity. Other problems include channel deformation, low solvent and acid/base resistivity, evaporation, and leaching. ${ }^{21}$ These limitations of PDMS have prompted researchers to look for alternative materials. ${ }^{13}$ PDMS can be fabricated by different methods such as casting, injection molding, imprinting, lithography, hot embossing, and laser ablation. The surface of the PDMS has to be modified using different methods such as plasma treatment, silanization, chemical vapor deposition, surfactants, graft polymer coating or using nanomaterial for the immobilization of biomarkers and further applications. ${ }^{21}$ PDMS based devices have been used in the detection of colorectal carcinoma, hepato cellular carcinoma, prostate cancer, bladder cancer, hepatitis B virus, dengue virus, meningitis and other food borne diseases. ${ }^{1}$

Thermoplastic platforms such as poly (methyl methacrylate) (PMMA), polycarbonate, polyester, and polyvinyl chloride are also widely used because of their chemical and mechanical properties. Unlike PDMS, it is compatible with most chemical reagents and biological assays, and gives better performance under mechanical stress. In addition, they are low-cost and easy to fabricate and massproduce. They can be fabricated by different methods such as hot embossing or imprinting, laser ablation, injection molding, and soft lithography. ${ }^{22}$

\section{Hybrid devices}

Each material has its own advantages and limitations as the microfluidic platform. Recently, there is a growing interest in hybrid device as a microfluidic platform. They take advantages of various platforms, while eliminating some limitations of certain platforms. They have been used for various applications. Zuo et al. ${ }^{23}$ developed a PDMS/paper hybrid microfluidic biochip. Paper acted as the platform for easy immobilization of nano-biosensors without complicated surface modification while PDMS provided the basic structure and glass provided the structural support. ${ }^{23}$ Dou et al. ${ }^{24}$ fabricated another PDMS/paper hybrid device for detection infectious diseases and multiplexed detection of meningitis. The hybrid device provided more stable performance than non-hybrid devices over a period of 2 months. ${ }^{24}$ Sanjay et al. ${ }^{9}$ developed miniaturized paper/PMMA hybrid microfluidic micro plate to complete immunoassay within an hour as compared to $18 \mathrm{~h}$ in traditional micro plates. Paper facilitated the rapid immobilization of protein and PMMA provided structural support and reagent delivery channels. ${ }^{9}$

\section{Conclusion}

There has been a tremendous increase in the trend to use lowcost substrate to develop a microfluidic device to perform an assay in resource-limited settings. These diagnostic devices have been enhanced due to improvement in automation and inclusion of functionalities. With the interfacing between material science, physics, biomedical engineering, chemistry, and biological sciences, the diagnostic devices are becoming simpler, sensitive, specific, and multifunctional. Smart phones are easy to use, inexpensive, and widely available. There is also an increasing trend to combine the diagnostic device with mobile phone based application, to be used in telemedicine, which can play a very important role to provide health services to people in underdeveloped countries. ${ }^{25,26}$ Although there are encouraging progresses in microfluidic devices, several limitations needs to be addresses before these devices can be used for routine diagnosis in the field. With all the progress in microfluidic devices, onchip sample pre-treatment, plasma separation, nucleic acid isolation and amplification still needs more attention and automation. Another issue to be addressed is the integration and automation of technology, specially the sample preparation and the detection methods. Many devices still use external complicated sample preparation and detection techniques. Fully integrated microfluidic device, which can achieve sample-in-answer-out capability will be the ultimate aim. These devices should be able to process crude real world sample such as blood and urine. Future devices should have the capability to include more clinical tests and trend should be to transform generalpurpose diagnostic device to personalized devices.

\section{Acknowledgements}

We are very grateful to School of Health and Allied Science, Pokhara University and Department of Microbiology, Pokhara Bigyan Tatha Prabidhi Campus for all their support.

\section{Conflict of interest}

The author declares no conflict of interest.

\section{References}

1. Sanjay ST, Fu G, Dou M, et al. Biomarker detection for disease diagnosis using cost-effective microfluidic platforms. Analyst. 2015;40(21):70627081 .

2. Sarnim G, Sanjay S, Roshan A, et al. Artemisia indica extracts as anthelminthic agent against Pheretima posthuma. Int J Pharm Pharm Sci. 2013;5(Suppl 3):259-262. 
3. Sarnim S, Jignesh S, Sanjay S, et al. Anthelminthic activity of Lavandula angustifolia Hidcote extracts using Pheretima posthuma. International Journal of Phytomedicine. 2012;4(1):22.

4. Bennett JE, Dolin R, Blaser MJ. Principles and practice of infectious diseases: Elsevier health sciences; 2014.

5. Hoskeri Y, Krishna V, Jignesh S, et al. In-silico drug designing using $\beta$-sitosterol isolated from Flaveria trinervia against peptide deformylase protein to hypothesize bactericidal effect. Int J Pharmacy Pharm Sci. 2012;4(2):192-196.

6. Ferlay J, Soerjomataram I, Dikshit R, et al. Cancer incidence and mortality worldwide: sources, methods and major patterns in GLOBOCAN 2012. Int J Cancer. 2015;136(5):E359-E386.

7. Yager P, Domingo GJ, Gerdes J. Point-of-care diagnostics for global health. Annu Rev Biomed Eng. 2008;10:107-144.

8. Dou M, Sanjay ST, Benhabib M, et al. Low-cost bioanalysis on paperbased and its hybrid microfluidic platforms. Talanta. 2015;145:43-54.

9. Sanjay ST, Dou M, Sun J, et al. A paper/polymer hybrid microfluidic microplate for rapid quantitative detection of multiple disease biomarkers. Scientific Reports. 2016.

10. Fu G, Sanjay ST, Dou M, et al. Nanoparticle-mediated photothermal effect enables a new method for quantitative biochemical analysis using a thermometer. Nanoscale. 2016;8(10):5422-5427.

11. Urdea M, Penny LA, Olmsted SS, et al. Requirements for high impact diagnostics in the developing world. Nature. 2006;444(Suppl 1):73-79.

12. Fu G, Sanjay ST, Li X. Cost-effective and sensitive colorimetric immunosensing using an iron oxide-to-Prussian blue nanoparticle conversion strategy. Analyst. 2016;141(12):3883-3889.

13. Sackmann EK, Fulton AL, Beebe DJ. The present and future role of microfluidics in biomedical research. Nature. 2014;507(7491):181-189.

14. Sanjay ST, Dou M, Fu G, et al. Controlled drug delivery using microdevices. Current pharmaceutical biotechnology. 2016;17(9):772_ 787.
15. Dou M, Sanjay ST, Dominguez DC, et al. Multiplexed instrument-free meningitis diagnosis on a polymer/paper hybrid microfluidic biochip. Biosens Bioelectron. 2017;87:865-873.

16. Nery EW, Kubota LT. Sensing approaches on paper-based devices: a review. Analytical and bioanalytical chemistry. 2013;405(24):75737595.

17. Hu J, Wang S, Wang L, et al. Advances in paper-based point-of-care diagnostics. Biosens Bioelectron. 2014;54:585-597.

18. Cheng CM, Martinez AW, Gong J, et al. Paper-Based ELISA. Angew Chem Int Ed Engl. 2010;49(28):4771-4774.

19. Cate DM, Adkins JA, Mettakoonpitak J, et al. Recent developments in paper-based microfluidic devices. Analytical chemistry. 2014;87(1):1941

20. Parolo C, Merkoçi A. Paper-based nanobiosensors for diagnostics. Chem Soc Rev. 2013;42(2):450-457.

21. Zhou J, Khodakov DA, Ellis AV, et al. Surface modification for PDMSbased microfluidic devices. Electrophoresis. 2012;33(1):89-104.

22. Tsao CW. Polymer Microfluidics: Simple, Low-cost fabrication process bridging academic lab research to commercialized production. Micromachines. 2016;7(12):225

23. Zuo P, Li X, Dominguez DC, Ye BC. A PDMS/paper/glass hybrid microfluidic biochip integrated with aptamer-functionalized graphene oxide nano-biosensors for one-step multiplexed pathogen detection. $L a b$ Chip. 2013;13(19):3921-3928.

24. Dou M, Dominguez DC, Li X, et al. A versatile PDMS/paper hybrid microfluidic platform for sensitive infectious disease diagnosis. Anal Chem. 2014;86(15):7978-7986.

25. Wac K. Smartphone as a personal, pervasive health informatics services platform: literature review. Yearb Med Inform. 2012;7:83-93.

26. Vashist SK, Mudanyali O, Schneider EM, et al. Cellphone-based devices for bioanalytical sciences. Analytical and bioanalytical chemistry. 2014;406(14):3263-3277. 